Revista Eletrônica de Ciência Administrativa (RECADM) - ISSN 1677-7387 Faculdade Cenecista de Campo Largo - Coordenação do Curso de Administração v. 2, n. 2, nov./2003 - http://revistas.facecla.com.br/index.php/recadm/

\title{
EL AVANCE DE LA INVESTIGACIÓN EN ESTRATEGIA EMPRESARIAL. UN ANÁLISIS COMPARADO ESPAÑA - CASTILLA Y LEÓN ${ }^{1}$
}

SANTOS ÁLVAREZ, Ma Valle

Profesora Titular de Universidad en Organización de Empresas

GARCÍA MERINO, Ma Teresa

Profesora Titular de Universidad en Organización de Empresas

MARTÍN CRUZ, Natalia

Profesora Titular de Universidad en Organización de Empresas

ORTEGA ÁLVAREZ, Ana

Profesora Titular de Escuela Universitaria en Organización de Empresas

Departamento. de Economía y Admón de Empresas, Universidad de Valladolid (España)

\section{RESUMEN}

El objetivo del trabajo es considerar el desarrollo alcanzado por el conocimiento en estrategia empresarial en Castilla y León y su posición en el conjunto nacional, ofreciendo una visión complementaria a la de anteriores estudios. Para dicho análisis se han considerado, durante la década de los 90 , congresos y revistas de alcance regional y nacional. Los resultados obtenidos nos muestran la necesidad de contar con más foros de encuentro en Castilla y León y con medios especializados en el campo de la estrategia. Además, los investigadores castellano-leoneses parecen mostrar un interés poco frecuente por la ejecución estratégica y por los aspectos de delimitación teórica, un tanto marginados en el conjunto nacional.

Palabras clave: Dirección estratégica, investigación, medios de difusión, España, Castilla y León.

ABSTRACT:

The aim of this research is to evaluate developments in strategic management knowledge within Castilla y León, comparing to the national ensemble and offering a complementary perspective to previous studies. We have considered for this analysis congresses and journals during the 90's in a regional and national scope. The results show two basic needs: to increase the number of workshops in Castilla y León and to provide specific resources for strategy analysis. Moreover, researchers in Castilla y León seem to have a special interest in regard to strategic execution and theoretical framework features, while those topics are far considered by national researchers.

Key words: Strategic management, research, media, Spain, Castilla y León.

\section{INTRODUCCIÓN.}

La dirección estratégica de la empresa (en adelante DEE) ha experimentado una transformación tan profunda en los últimos tiempos, que, no es de extrañar, que se diga de ella que cuenta con un cuerpo de conocimiento propio (Ventura, 1996, p.11; Fernández, 1999, p.11). Su desarrollo se ha visto favorecido por el avance del propio campo de estudio -propiciado, claro está, por el progreso logrado en áreas empresariales como las de finanzas, marketing u operaciones- y de las vías utilizadas para darle difusión (Franke et al., 1990, p.252 y Rumelt et al., 1994, pp.12-13). Publicaciones periódicas, congresos y demás puntos de encuentro han sido, por tanto, decisivos para el progreso de la DEE, al servir como indicador del grado de desarrollo alcanzado por la disciplina y como espejo donde se reflejan las cuestiones centrales en cada momento. Respecto a dichos medios queremos matizar que, tal y como se plantea en Guerras et al. (1999) y en García et al (1999b), cabe pensar que el proceso normalmente seguido por los investigadores para la difusión de sus trabajos conste de dos etapas. En la primera, los trabajos se presentan en Congresos $u$ otros encuentros científicos similares, en los que se recibe una respuesta inmediata de un

\footnotetext{
${ }^{1}$ Este trabajo ofrece una síntesis del proyecto titulado Estado de la investigación en estrategia empresarial en Castilla y León, que fue objeto de subvención por la Consejería de Economía y Hacienda de la Junta de Castilla y León (Orden de 28 de junio de 1999). Una versión previa del mismo se presentó en el $7^{\circ}$ Congreso de Economía Regional de Castilla y León (Soria, noviembre de 2000).
} 
número de receptores no siempre elevado. Una vez atendidas las sugerencias recogidas en esta fase, en la siguiente los trabajos se remiten a alguna publicación apropiada y, si es posible, relevante para el campo de estudio. De este modo, siempre que se supere el proceso de evaluación, los resultados de la investigación gozarán de un amplio alcance, al llegar -en un tiempo normalmente prolongado- a un elevado número de lectores.

Con objeto de determinar en qué medida ha avanzado el conocimiento en DEE y en qué dirección lo ha hecho, se han realizado una serie de investigaciones consistentes en analizar la posición de la DEE española en el ámbito internacional y la senda seguida por la investigación española sobre estrategia desde mediados de los años ochenta (García et al. 1999a, 1999b y 2000; García y Santos, 2000b; Santos et al. 1999a y b). El objetivo del presente estudio es detenerse a considerar el desarrollo alcanzado por el conocimiento en DEE en Castilla y León y su posición en el conjunto nacional, ofreciendo así una visión complementaria a la proporcionada en los citados trabajos. Para efectuar dicho análisis optamos por considerar aquellos congresos y revistas de alcance regional ${ }^{2}$ y nacional en los que hayan podido tener cabida trabajos sobre estrategia empresarial. Hemos decidido abordar ambas vías de difusión -congresos y revistas- con objeto de que nuestro análisis sea lo más completo posible -teniendo en cuenta que puede haber investigadores que únicamente utilicen una de las dos vías- y con el propósito de considerar esa secuencia que antes hemos descrito para el proceso de difusión de la investigación. Por lo que respecta al tiempo, la propia evolución y desarrollo de este campo de conocimiento nos ha llevado a centrar nuestro análisis en un periodo lo más cercano posible: 1990-98 para los congresos y 1990-97 para las revistas ${ }^{3}$. Se trata de un intervalo lo suficientemente amplio y reciente como para que sea representativo de la evolución y estado actual del campo objeto de estudio y proporcione, además, una garantía de calidad, debido a la generalización, en esta década, de procesos de evaluación y selección cada vez más rigurosos en los diversos medios de difusión.

La realización de un análisis como el que aquí se propone permite desvelar aspectos de gran interés, tanto para los investigadores como para los profesionales de empresa, los poderes públicos y la sociedad en general. A los investigadores les permite conocer qué parcelas del campo de la DEE han sido insuficientemente tratadas y reclaman, por tanto, una mayor atención por su parte; quiénes investigan sobre cada tema, de modo que se pueda facilitar la coordinación en el estudio y, en definitiva, el ahorro de esfuerzos; por dónde se canaliza preferentemente la difusión de los resultados de la investigación; dónde busca el investigador su referente teórico -dentro o fuera del ámbito español-; qué avances se han logrado a lo largo del tiempo, y, cómo no, cuáles son los logros particulares alcanzados en el ámbito de Castilla y León y en qué medida los investigadores castellano-leoneses han aunado esfuerzos para evitar duplicidades. Por su parte, las empresas, el gobierno y la administración de Castilla y León dispondrán así de alguna información acerca de los sujetos que centran la atención de sus investigadores en el campo de la DEE; de la escasez o disponibilidad de medios adecuados para dar a conocer los resultados de dicha investigación -sea a otros investigadores, a profesionales de la empresa o a la sociedad en general-; del grado de coordinación existente entre los distintos centros investigadores, y, en definitiva, del uso de los recursos destinados a la investigación en DEE en su correspondiente ámbito de referencia y de los resultados alcanzados con tales recursos.

\footnotetext{
${ }^{2}$ Como es lógico, atendiendo al objetivo del trabajo, únicamente vamos a considerar aquí Castilla y León.
} 
Revista Eletrônica de Ciência Administrativa (RECADM) - ISSN 1677-7387

Faculdade Cenecista de Campo Largo - Coordenação do Curso de Administração

v. 2, n. 2, nov./2003 - http://revistas.facecla.com.br/index.php/recadm/

Presentado nuestro objetivo y efectuadas algunas consideraciones previas, pasamos ahora a señalar la estructura del trabajo. En el epígrafe que sigue a esta introducción vamos a presentar brevemente cuál ha sido la evolución de los medios de difusión que acogen trabajos sobre estrategia empresarial. Tendremos en cuenta para ello los dos marcos espaciales considerados en el análisis: España y Castilla y León. El tercer epígrafe nos lleva a centrarnos en la otra vertiente de desarrollo de un campo científico -la investigación-, respecto a la que describiremos cuál ha sido el proceso de análisis aquí seguido y cuáles son los resultados alcanzados. Por último, en el epígrafe final, comentaremos las conclusiones más relevantes extraídas de la investigación.

\section{EVOLUCIÓN EN MEDIOS DE DIFUSIÓN Y LA ESTRATEGIA EMPRESARIAL.}

La DEE, que emerge como disciplina hace más de cuarenta años y ha recibido aportaciones tanto del mundo académico como del ámbito empresarial, es hoy día una materia académica y un campo de análisis respetado y con una cierta entidad (Snow y Thomas, 1994, p.474). Presenta un carácter pluralista, dado que no cuenta con un paradigma dominante sino con el soporte de tres paradigmas complementarios (Mathé, 1995, pp.40-41; Teece et al., 1997, p.510) y recurre al empleo de una pluralidad de métodos para el desarrollo de la investigación (Snow y Thomas, 1994). En la evolución histórica de la DEE podemos identificar tres grandes etapas (García et al., 1999b): nacimiento (década de los sesenta), desarrollo (años setenta y ochenta) y consolidación (coincidiendo básicamente con los noventa). En España, el germen de la DEE -o por aquél entonces política de empresa- como disciplina académica podría situarse en los años cincuenta, con la creación de la sección de Economía de la Empresa en la Licenciatura de Económicas (Salas, 1995, p.815). En el ámbito de Castilla y León, es la Universidad de Valladolid la primera que cuenta con Facultad de Ciencias Económicas y Empresariales -desde mediados de los años 70- y la primera, por tanto, en impartir dicha disciplina. Se sientan por aquel entonces las bases para un desarrollo de la investigación sobre estrategia en tales espacios de referencia.

Paralelamente al desarrollo de la DEE, se ha producido la evolución de una serie de medios utilizados para su difusión. En los países anglosajones, los esfuerzos iniciales por organizar sociedades y publicaciones se ven influidos por el interés que, tras la aparición del trabajo de Ansoff, despierta el fenómeno de la planificación. Se entiende así que los primeros trabajos en estrategia encuentren su medio de difusión en revistas tales como Long Range Planning y The Planning Review. Las revistas generales sobre dirección completan los medios de difusión del momento. Esta situación se prolonga hasta la década de los ochenta, cuando se crea la Strategic Management Society y emergen dos revistas especializadas: Strategic Management Journal y The Journal of Business Strategy, la primera orientada a la investigación académica y la segunda a la práctica. A ellas se han unido a lo largo del tiempo otras publicaciones de carácter más general, alcanzando diferente prestigio y notoriedad en el ámbito académico. En la actualidad, dos son las revistas que gozan de mayor reconocimiento entre los especialistas académicos en estrategia: Strategic Management Journal y Academy of Management Review.

En el ámbito español, hay que esperar casi hasta los años ochenta para que se constituyan ciertas asociaciones en las que se dé cabida a los investigadores de la estrategia empresarial y con las que se

\footnotetext{
${ }^{3}$ Ya que algunas revistas cuentan con cierto retraso en la presentación de sus números, hemos preferido considerar periodos temporales diferentes para congresos y revistas.
} 
propicie el encuentro y el intercambio de conocimiento de éstos (tabla 1). Conviene destacar, sin embargo, que, tanto en el conjunto nacional como en Castilla y León, carecemos de foros de encuentro especializados en DEE. Puede decirse entonces que, como en el mundo anglosajón, los investigadores españoles en DEE difunden en buena medida el fruto de sus trabajos en congresos de carácter general -ya sea en economía, en economía de empresa o en el ámbito de la dirección-, pero que, a diferencia de ellos, hasta la fecha no contamos con ningún foro reconocido y consolidado que se haya especializado en estrategia. Estos aspectos se perciben de forma incluso más acusada en el contexto castellano-leonés, donde sólo contamos con el Congreso de Economía Regional de Castilla y León -de carácter general y periodicidad bianual-. Aunque, en principio, podría tener aquí cabida cualquier otro tipo de encuentro o grupo de trabajo de alcance nacional o regional que, de forma directa o indirecta, girase en torno a la DEE, finalmente han pesado aquí, para no incluirlos, razones de permanencia, difusión y reconocimiento, entre otras.

En cuanto a las publicaciones periódicas, conviene señalar una serie de aspectos que, ampliamente expuestos en García et al. (1999a y 2000) y en Santos et al. (1999b), recogemos a continuación brevemente. No existen en nuestro país revistas especializadas en estrategia, de modo que los investigadores se ven obligados a compartir medios de difusión con los de otros campos científicos, bien sea de economía de la empresa o de economía. Contamos con un foro de publicaciones reconocidas entre los investigadores en estrategia ${ }^{4}$, entre las que coexisten revistas de larga tradición con otras mucho más jóvenes -pues no en vano en los noventa se concentra el mayor número de nuevas publicaciones al respecto-. Además, resulta curioso advertir que las revistas en las que la estrategia parece contar con mayor peso se encuentran sobre todo entre las creadas en los años 90, lo que permite plantear que es a partir de esa década cuando empieza a irse delimitando, de forma precisa, el ámbito de encuentro de los investigadores en DEE por lo que a las revistas se refiere. También nos parece interesante señalar que algunas de las revistas que cuentan con mayor peso de la estrategia han sido promovidas por algunas de las asociaciones más arriba mencionadas. Finalmente, y por lo que respecta a las publicaciones en el ámbito de Castilla y León, también tendríamos que hablar de una carencia de revistas especializadas en estrategia. $\mathrm{Y}$ de escasez cuando las que consideramos son las publicaciones de carácter general en dirección, en economía de empresa o en economía. No obstante, conviene destacar la existencia de alguna publicación promovida desde el ámbito universitario y el esfuerzo desarrollado recientemente por algunas instituciones para la puesta en marcha de otras nuevas. Así, por ejemplo, la Revista de Economía y Finanzas de Castilla y León -con su primer número en 1998- o la Revista de Investigación Económica y Social de Castilla y León, cuyo primer número es del primer semestre de 1999.

\begin{tabular}{lcccc}
\hline $\begin{array}{c}\text { ENTIDAD } \\
\text { ORGANIZADORA }\end{array}$ & $\begin{array}{c}\text { AÑO DE } \\
\text { CREACIÓN }\end{array}$ & $\begin{array}{c}\text { DENOMINACIÓN } \\
\text { DEL CONGRESO }\end{array}$ & $\begin{array}{c}\text { PRIMER } \\
\text { CONGRESO }\end{array}$ & PERIODICIDAD \\
\hline $\mathrm{AECA}^{5}$ & 1979 & AECA & 1981 & Bianual \\
$\mathrm{AEDEM}^{6}$ & Mediados 80 & AEDEM & $\begin{array}{c}1987 \text { (Actas } \\
\text { desde } 1993)\end{array}$ & Anual \\
$\mathrm{ACEDE}^{7}$ & 1990 & ACEDE & 1991 (Actas & Anual \\
\hline
\end{tabular}

\footnotetext{
${ }^{4}$ Ver anexo 1.

${ }^{5}$ Asociación Española de Contabilidad y Administración de Empresas.

6 Asociación Española de Dirección y Economía de Empresa. Desde 1992 se integra en la Asociación Europea de Dirección y Economía de Empresa.
} 
Revista Eletrônica de Ciência Administrativa (RECADM) - ISSN 1677-7387

Faculdade Cenecista de Campo Largo - Coordenação do Curso de Administração

v. 2, n. 2, nov./2003 - http://revistas.facecla.com.br/index.php/recadm/

\begin{tabular}{|c|c|c|c|c|}
\hline & & & desde 1995) & \\
\hline Junta de Castilla y León & -- & $\begin{array}{c}\text { CONGRESO DE } \\
\text { ECONOMÍA } \\
\text { REGIONAL DE } \\
\text { CASTILLA Y LEÓN }\end{array}$ & 1988 & Bianual \\
\hline $\begin{array}{l}\text { Consejo General de } \\
\text { Colegios de Economistas } \\
\text { de España }\end{array}$ & 1971 & $\begin{array}{l}\text { CONGRESO } \\
\text { NACIONAL DE } \\
\text { ECONOMÍA }\end{array}$ & 1982 & Trianual \\
\hline
\end{tabular}

Tabla 1: Asociaciones y entidades promotoras de encuentros en los que tiene cabida la DEE.

Fuente: Elaboración propia a partir de la información suministrada por las diferentes

entidades consideradas.

\section{ANÁLISIS COMPARADO ESPAÑA - CASTILLA Y LEÓN PARA LA INVESTIGACIÓN EN ESTRATEGIA EMPRESARIAL.}

Presentados ya los medios de difusión, conviene ahora que nos centremos en mostrar cuál ha sido el análisis que hemos realizado en torno a la investigación sobre estrategia en España y en Castilla y León y qué resultados hemos alcanzado.

\subsection{Descripción del proceso de análisis.}

El estudio del esfuerzo investigador desarrollado en un campo científico exige disponer de una precisa delimitación de dicho campo. Si además el análisis pasa por la revisión de distintos medios de difusión, se hace preciso acotar también la relación de medios -foros de debate y publicaciones- a tener en cuenta. En el caso de la DEE, la diversidad y pluralidad de este ámbito hacen compleja su delimitación (Castagnos et al.,1997, p.40; Martinet, 1993, p.72; Mathé, 1995, p.32 y García y Santos, 2000a) y la dispersión en los medios de difusión utilizados por los investigadores dificulta la tarea de precisar la relación de aquellos que van a ser aquí objeto de análisis. Investigaciones previas nos permiten disponer, con ciertas garantías, de esos elementos e iniciar el estudio planteado. Así, contamos ya con un listado de términos clave en $\mathrm{DEE}^{8}$ (García et al., 1999a), que, agrupados en cinco bloques temáticos, ha sido definido por especialistas académicos en dicho ámbito y nos muestra la amplitud y diversidad del campo considerado. Igualmente, la definición de ese foro de publicaciones españolas relevantes en DEE al que aludíamos en el epígrafe anterior, nos proporciona un soporte riguroso para determinar las revistas que queremos considerar en el estudio. Tenemos que matizar que no todas las publicaciones que componen dicho foro han sido objeto de revisión en el análisis aquí propuesto. En este sentido, hemos prescindido de las revistas Cuadernos de Economía y Dirección de Empresa (CEDE) y Esic-Market. La juventud de la revista nos ha llevado a tomar tal decisión en el primer caso -su primer número es del año 1998- y para Esic-Market, a pesar de ser ampliamente conocida y de obtener una puntuación favorable en el proceso de determinación del foro, consideramos que mantiene una muy estrecha relación con el área de marketing. En contraposición, hemos decidido incorporar al grupo, con carácter temporal, la revista Anales de Estudios Económicos y Empresariales, por ser representativa del espacio castellano-leonés $y$, al tiempo, por su proximidad para los autores de esta investigación ${ }^{9}$. Habrá, no obstante, que ser cautos a la hora de interpretar los resultados, debido a las distorsiones que la incorporación de ésta última pudiera generar. En lo referente a qué congresos considerar, hemos recurrido a esa relación de los principales encuentros a

\footnotetext{
${ }^{7}$ Asociación Científica de Economía y Dirección de Empresa.

${ }^{8}$ Ver Anexo 2.

${ }^{9}$ No hemos considerado otras revistas del ámbito de Castilla y León citadas con anterioridad, por ser, como se ha visto, de reciente aparición.
} 
nivel nacional y regional que aparece recogida en la tabla 1. Únicamente señalaremos que las dificultades encontradas para disponer de las actas de los congresos de AECA y, al mismo tiempo, su mayor orientación hacia el campo contable nos han llevado a prescindir de este foro de debate en el presente análisis.

La relación de medios de difusión que son aquí objeto de consideración queda, por tanto, integrada por cuatro congresos y trece publicaciones. A partir de ahí, y con objeto de llevar a cabo el estudio planteado, hemos revisado los trabajos incluidos en los medios de difusión que se acaban de señalar para los intervalos de tiempo en cada caso especificados. De este modo, hemos contado con dos bases de datos -una para congresos y otra para revistas- en las que, junto a otros, se han incluido los siguientes registros: título del trabajo; autores, con su centro de trabajo; citas asociadas ${ }^{10}$, como expresión del objetivo perseguido o de las principales cuestiones abordadas en el mismo; año y congreso o revista en la que aparece. En el caso de los congresos recogemos, además, el número de referencias bibliográficas utilizadas en cada comunicación, distinguiendo por idioma y señalando también cuántas de ellas corresponden a aquellas publicaciones con mayor reconocimiento en los foros anglosajón ${ }^{11}$ y español respectivamente. Tanto la selección de trabajos como la asignación de citas se han realizado en función del título del trabajo, de su resumen y de su autor o autores. Sólo cuando dichos indicadores no resultaban suficientes hemos recurrido al análisis del contenido del trabajo.

\subsection{Investigación sobre DEE en congresos (1990-1998). Análisis para España y Castilla y León.}

A partir de las actas disponibles en el periodo 1990-1998 para los cuatro congresos considerados, hemos elaborado una base de datos formada por 389 trabajos. De ellos, 34 corresponden a investigadores de Castilla y León (en adelante CYL). Así pues, de los trabajos que, sobre estrategia, se han presentado en el conjunto de congresos analizados, en torno a un $9 \%$ han sido elaborados por investigadores de la comunidad castellano-leonesa. Cualquier comentario en torno a dicho porcentaje únicamente debería hacerse si se dispusiese de información comparada acerca de, entre otros aspectos, el número de investigadores y el volumen de recursos disponibles en los espacios geográficos considerados. Nos interesa asimismo destacar el elevado número de citas -633 en España y 59 en CYL- con que contamos para el conjunto de trabajos seleccionados, de manera que podemos decir que a cada trabajo se le asignan por término medio 1.63 y 1.73 citas en España y CYL respectivamente. Cabría entonces decir que la investigación en DEE, tanto en el conjunto de España como en nuestra Comunidad Autónoma, no se plasma en trabajos centrados en una única cuestión temática, sino que más bien recurre a una óptica plural con la que se repara, al mismo tiempo, en cuestiones diferentes a aquélla que constituye el objeto central de análisis. Lo que parece lógico si tenemos en cuenta que, tal y como señalan Johnson y Scholes (1997, p.14), la DEE no se desarrolla de forma lineal sino que más bien sus elementos están concatenados.

Si ahora observamos cómo se distribuyen tales trabajos a lo largo del periodo temporal estudiado (figura 1), podemos señalar que la presencia significativa de trabajos sobre estrategia empresarial se

10 En adelante utilizamos esta denominación para referirnos a la asignación que hacemos, para cada uno de los trabajos seleccionados, de las palabras clave incluidas en ese listado de términos clave al que antes hemos aludido (anexo 2).

${ }^{11}$ El foro anglosajón y su delimitación están claramente presentados en Santos et al. (1999a) y en García et al. (1999b). 
produce a partir de 1994 ó 1995. Podrían proporcionarse dos explicaciones al respecto: un cierto retraso en la celebración de los primeros encuentros con un carácter más específico en dirección y economía de empresa $^{12}$-pues no hay que olvidar que los otros se dirigen a abarcar el conjunto de la economía- $y$, sobre todo, un relativo desfase temporal en la elaboración de actas para dichos congresos ${ }^{13}$. La figura 1 indica, además, que frente a la irregular e interrumpida presencia de los trabajos de CYL en el tiempo, la tendencia del conjunto de investigadores españoles parece ser de continuo crecimiento, que empezaría quizá a estabilizarse al final del periodo. A nuestro juicio, dicha estabilización podría asociarse al comienzo de la etapa de madurez en DEE, quizá iniciada en España con un cierto retraso, pero también a una creciente inquietud entre los investigadores en DEE por tratar de difundir el fruto de sus esfuerzos en el ámbito internacional, lo que podría mermar en ocasiones su presencia en encuentros de ámbito nacional o regional.

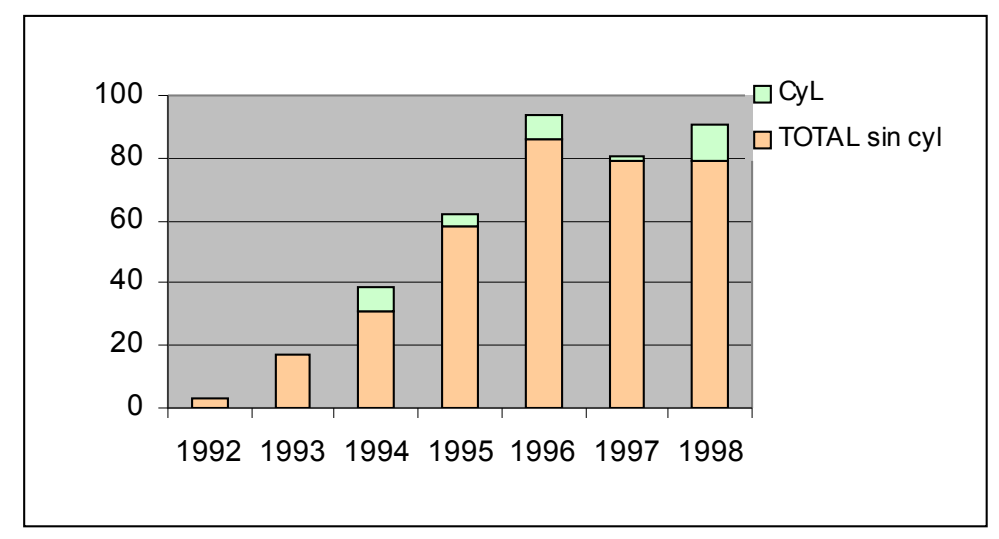

Figura 1: Evolución temporal de la DEE en congresos. El lugar de CYL.

Efectuada esta primera aproximación a la base de datos, a continuación presentamos lo que de la misma se desprende en relación con los siguientes aspectos: congresos analizados, centros de trabajo, perfil temático y referentes bibliográficos. Abordaremos cada uno de ellos desde una doble óptica de análisis (estática y evolutiva) e insistiremos en la comparación España-CYL.

Congresos analizados: Tal y como puede verse en la figura 2, los datos muestran desequilibrios significativos por lo que respecta al peso de los distintos encuentros considerados. Así, se constata que, ante la ausencia de foros especializados en DEE, los investigadores prefieren acudir, para la difusión de sus logros, a congresos propios de la dirección y economía de empresa y sólo en muy contadas ocasiones a congresos de economía. De este modo, del total de trabajos de DEE presentados en congresos durante el periodo estudiado, la mayoría (el 91\%) se defendieron en AEDEM y en ACEDE. Algo superior resulta la presencia en AEDEM, aunque debe tenerse en cuenta la posterior creación de ACEDE y, sobre todo, la tardía disponibilidad de actas para el caso de este congreso.

En Castilla y León se mantienen esos desequilibrios, pero se observan ciertas peculiaridades. Por ejemplo, que el porcentaje de trabajos presentados en AEDEM y ACEDE se sitúa bastante por detrás del nacional -un $65 \%$ frente al $91 \%$ antes señalado-; que aquí el Congreso de Economía Regional tiene un peso ciertamente relevante (33\%), situándose incluso por delante de uno de los congresos específicos en dirección y economía de empresa (AEDEM); y que, a diferencia del conjunto nacional, ACEDE se sitúa a la

\footnotetext{
${ }^{12}$ El primer congreso de AEDEM tuvo lugar en 1987 y en 1991 el primero de ACEDE.
} 
cabeza. Parece entonces que a los investigadores castellano-leoneses les atraiga el foro propio de su comunidad y que, de los congresos de dirección, tiendan a decantarse por aquél que, como su misma denominación recoge, tiene un claro carácter científico.

Por lo que respecta a la evolución temporal del peso relativo de los distintos congresos, podemos indicar una continuada presencia de los investigadores de DEE en los congresos de AEDEM y ACEDE frente a la presencia algo más irregular en los dos restantes ${ }^{14}$-, mostrando, en general, una tendencia creciente en el conjunto nacional, que no se percibe de forma tan clara cuando nos circunscribimos a CYL, donde se aprecian más oscilaciones.

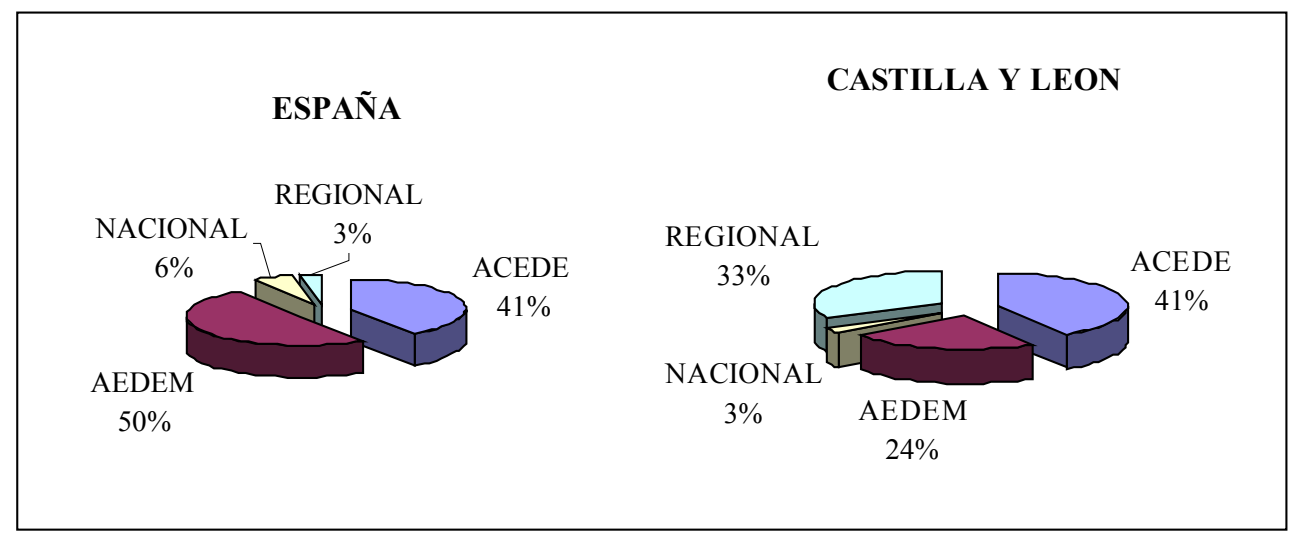

Figura 2: Peso relativo de trabajos sobre DEE por congresos.

Centros de trabajo: Nos referimos ahora a la ubicación de los autores de los trabajos en las distintas universidades españolas, centros de investigación u otras organizaciones. La tabla 2 nos permite resumir la información más relevante que, a este respecto, nos proporciona la base de datos. Podemos señalar, en primer lugar, que, del total de centros presentes en la base, la mayoría son universitarios (un 78\%, con un $95 \%$ de las citas asociadas), lo que resulta ser menos acentuado cuando nos referimos a $\mathrm{CYL}^{15}$ (un $57 \%$, con un $92 \%$ de las referencias). En segundo lugar destaca, para el periodo considerado, la escasa colaboración entre centros -muy baja cuando se trata del conjunto nacional (sólo en un $9 \%$ del total de 389 trabajos) y algo menos reducida cuando nos referimos a CYL (en torno a un 20\% de sus 34 trabajos)-, lo que explica entonces que a cada trabajo se le asignen, por término medio, 1.1 ó 1.2 centros de trabajo, según se trate de España o de CYL. Los datos recogidos revelan, en tercer lugar, que la cooperación entre países es muy poco significativa -un 3\% para España y casi un 6\% en CYL-, lo que permite explicar esos promedios de 1.035 y 1.11 países por trabajo respectivamente.

\footnotetext{
${ }_{14}^{13}$ Así, las primeras actas disponibles de AEDEM son del año 1993 y las primeras de ACEDE de 1995.

14 Téngase en cuenta, no obstante, el carácter bianual del Congreso de Economía Regional de CYL y la celebración cada tres años del Congreso Nacional de Economía.

${ }_{15}$ Quizá debido a que, a diferencia de los otros congresos, existe toda una diversidad de centros universitarios y, sobre todo, no universitarios- con participación en el Congreso de Economía Regional de Castilla y León.
} 
Revista Eletrônica de Ciência Administrativa (RECADM) - ISSN 1677-7387 Faculdade Cenecista de Campo Largo - Coordenação do Curso de Administração v. 2, n. 2, nov./2003 - http://revistas.facecla.com.br/index.php/recadm/

\begin{tabular}{|lcc|}
\hline & TOTAL & CASTILLA Y LEON \\
\hline $\mathrm{N}^{\circ}$ centros & 72 & 7 \\
\hline $\mathrm{N}^{0}$ c.universitarios & 56 & 4 \\
\hline $\mathrm{N}^{\circ}$ c.no universitarios & 16 & 3 \\
\hline $\mathrm{N}^{\circ}$ trabajos & 389 & 34 \\
\hline Trabajos con 3 centros & 3 & 0 \\
\hline Trabajos con 2 centros & 33 & 7 \\
\hline Trabajos con 3 paises & 1 & 0 \\
\hline Trabajos con 2 países & 12 & 2 \\
\hline
\end{tabular}

- TOTAL:
1.10 Centros por trabajo
1.035 Países por trabajo
- CASTILLA Y LEON
1.2 Centros por trabajo
1.11 Países por trabajo

Tabla 2: Trabajos de DEE por tipos de centros y trabajos en equipo.

Si nos detenemos ahora en la distribución individualizada por centros de los diferentes trabajos que conforman esta base de datos, tendríamos que constatar, de entrada, la escasa participación de organizaciones no universitarias (de sólo un 5\%) y el desequilibrio existente en la participación de las distintas universidades (figura 3). Así, mientras a la Universidad de Oviedo le corresponde la autoría de un $17 \%$ de los trabajos, las cuatro universidades castellano-leonesas no alcanzan en conjunto más que un $7 \%$. Excesiva juventud en algunos casos, lentitud o dificultades en la consolidación de ciertos equipos de investigación, volumen de recursos disponibles -ya sean humanos, financieros, etc.-, escasa atención a la investigación en DEE, podrían ser, junto a otras, algunas de las razones a barajar para justificar tales diferencias. En cualquier caso, conviene precisar que, por lo que a CYL se refiere, a la Universidad de Valladolid se debe el $50 \%$ de los trabajos, un 29\% a la Universidad de Salamanca, un 12\% a la de León y, por último, a la joven Universidad de Burgos -creada por Ley 12/1994, de 26 de mayo- un 6\%. Así, la Universidad de Valladolid parecería ir a la cabeza de la investigación sobre estrategia en la comunidad castellano-leonesa, no sólo por el número de trabajos presentados en congresos sino también por la continuidad de su participación.

\section{DISTRIBUCIÓN POR CENTROS}

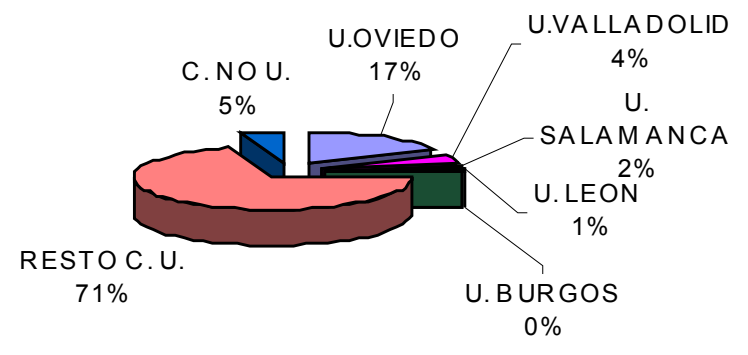

Figura 3: Distribución de los trabajos en congresos por centros. El lugar de CyL

Perfil temático: Se trata, en este caso, de detenernos a mostrar cuáles son los tópicos y las cuestiones que centran el interés de los investigadores en este campo. Para ello nos hemos fijado en las palabras clave asignadas a cada trabajo. La consideración de las figuras 4 y 5 denota la existencia de un claro desequilibrio en el esfuerzo investigador dedicado a los distintos bloques temáticos que integran la 
DEE. Así, vemos que mientras el bloque dedicado a la ejecución estratégica abarca únicamente un 5\%, el relativo a objetivos, formulación y elección de la estrategia copa el $43 \%$ de las citas asignadas en España y el $58 \%$ de las citas en CYL. Para los bloques restantes podríamos decir que, en ambos casos, se aprecia cierta homogeneidad en cuanto a la atención recibida, aunque menos clara en el conjunto de España que en CYL. Curioso puede resultar observar que, mientras en CYL la atención dedicada al análisis estratégico interno es ligeramente superior al análisis externo (del sector y de la competencia), en el conjunto de España aún sucede al contrario. Y ello a pesar de que los investigadores en DEE han tomado conciencia desde finales de los años 80 y gracias al enfoque de recursos y capacidades- de la importancia de desarrollar un adecuado análisis estratégico interno. Si prestamos ahora atención a las diferentes palabras clave, podemos decir que las desigualdades se mantienen y que, tanto en España como en CYL, los temas del desarrollo cooperativo y el desarrollo de mercados (intemacionalización) ocupan las posiciones más destacadas. Ahora bien, el orden de interés no es el mismo en ambos casos. Así, los temas que concentran mayor atención en la investigación española son: desarrollo cooperativo y desarrollo de mercados. Detrás, pero con una cierta distancia, tendríamos: estrategia y proceso estratégico, caracterización del sector, recursos y capacidades, ventaja competitiva y entorno general. En el caso de CYL, los que ocupan las primeras posiciones son: objetivos, desarrollo de mercados, desarrollo cooperativo y entorno general. Y, por lo que se refiere a las cuestiones que no parecen haber despertado interés entre los investigadores o éste ha sido muy reducido, podemos citar las siguientes: carteras de negocio, reputación y legitimidad, stakeholders, profundización, diversificación horizontal, valoración y evaluación estratégica, selección de la estrategia o planificación y control estratégico. Aunque con ciertas diferencias, los estudiosos de CYL no parecen disentir del conjunto a este respecto.
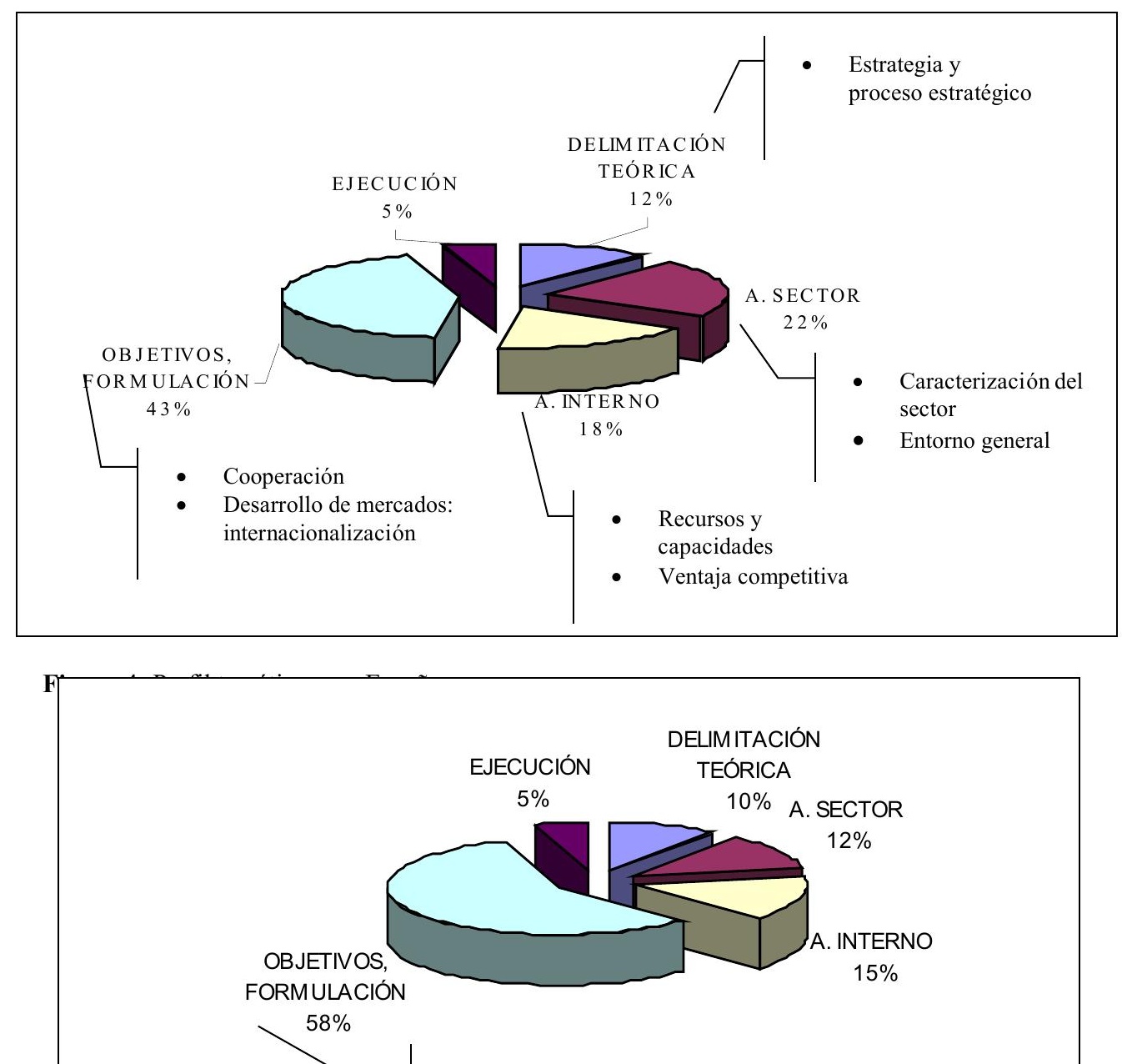
Revista Eletrônica de Ciência Administrativa (RECADM) - ISSN 1677-7387

Faculdade Cenecista de Campo Largo - Coordenação do Curso de Administração

v. 2, n. 2, nov./2003 - http://revistas.facecla.com.br/index.php/recadm/

Por último, si consideramos de forma evolutiva el perfil temático podríamos hablar de una clara continuidad en el conjunto de la investigación española -al menos para los términos clave que despiertan mayor preocupación-, que no encontramos, sin embargo, en CYL. ¿Cabría pensar, entonces, que los investigadores castellano-leoneses dispersamos nuestra atención por diferentes sujetos y que apenas nos centramos de forma continuada en ninguno? Sería ciertamente arriesgado hacer una afirmación de tal tipo, pues sólo podría sostenerse si esto se confirmara con el análisis de las revistas y con otros análisis complementarios -de proyectos de investigación, de tesis defendidas, etc.-.

Referentes bibliográficos: Tan sólo nos queda ya aludir a las referencias bibliográficas que sirven de soporte a los trabajos revisados en esta base de datos. Tanto para el total como para el caso de CYL encontramos un claro liderazgo de las referencias bibliográficas en inglés, seguidas a cierta distancia por las españolas y sólo muy de lejos por referencias en otros idiomas (tabla 3), dejando claro el dominio anglosajón en el campo de la DEE. Para confirmar este dominio, y dado que investigadores de centros no anglosajones podrían perfectamente escribir en inglés, hemos completado el análisis de las referencias bibliográficas determinando si corresponden a alguna de las publicaciones consideradas más prestigiosas en el foro anglosajón apreciado en estrategia (Santos et al., 1999a; García et al., 1999b) ${ }^{16}$. Para el caso de las referencias en español también hemos completado el análisis, determinando si corresponden a alguna de las publicaciones pertenecientes al grupo que, como puede verse en García et al. (1999a y 2000, p.83), cuenta con un reconocimiento académico más elevado dentro del foro español apreciado en estrategia. A éstas hemos añadido la Revista Europea de Dirección y Economía de la Empresa, pues, aunque pertenece al grupo de reconocimiento medio, es la que, como se pone de manifiesto en García et al. (1999a y 2000, p.87), parece contar con mayor peso de la DEE. Los resultados de este análisis son claros: el liderazgo lo ejerce -como era de esperar- la Strategic Management Journal, de forma incluso más acentuada en CYL (7,25 de promedio) que en el conjunto nacional (4,52); le siguen las Academys y la Administrative Science Quarterly, con un peso similar en el total y en CYL; a una cierta distancia se sitúan las referencias del foro español, que, tanto para el total como para CYL, presentan el mismo orden de preferencia en las de mayor peso-Papeles de Economía Española, Información Comercial Española, Economía Industrial- y un orden diferente en el resto. Vemos así que la Revista Europea de Dirección y Economía de la Empresa se encuentra entre las menos citadas. Y una última información que se desprende de la tabla 3: en los trabajos de investigación de CYL presentados a

${ }^{16}$ En estos trabajos puede verse cuáles son las publicaciones que integran el foro anglosajón de revistas apreciadas en DEE y, de entre ellas, cuáles son las más prestigiosas. 
Revista Eletrônica de Ciência Administrativa (RECADM) - ISSN 1677-7387 Faculdade Cenecista de Campo Largo - Coordenação do Curso de Administração v. 2, n. 2, nov./2003 - http://revistas.facecla.com.br/index.php/recadm/ congresos parece citarse menos que en el conjunto nacional (un promedio de 22,64 frente al 24,39 del total).

\begin{tabular}{|c|c|c|c|c|c|c|}
\hline & \multicolumn{3}{|c|}{ ESPAÑA } & \multicolumn{3}{c|}{ CASTILLA-LEÓN } \\
\hline Ref-inglés & MEDIO & MAX. & MIN. & MEDIO & MAX. & MIN. \\
\hline Ref-francés & 10,06 & 117 & 0 & 8,41 & 98 & 0 \\
\hline Ref-español & 0,44 & 28 & 0 & 0,36 & 8 & 0 \\
\hline Ref-otros idiomas & 6,09 & 41 & 0 & 6,94 & 41 & 0 \\
\hline Total referencias & 0,10 & 9 & 0 & 0 & 0 & 0 \\
\hline Academy of Management Review & $\mathbf{2 4 , 3 9}$ & $\mathbf{1 2 1}$ & $\mathbf{0}$ & $\mathbf{2 2 , 6 4}$ & $\mathbf{1 0 1}$ & $\mathbf{6}$ \\
\hline Strategic Management Journal & 4,52 & 10 & 0 & 2,33 & 3 & 1 \\
\hline Academy of Management Journal & 2,43 & 18 & 0 & 2,25 & 4 & 1 \\
\hline Administrative Science Quarterly & 2,18 & 9 & 0 & 2 & 3 & 1 \\
\hline Papeles de Economía Española & 0,38 & 11 & 0 & 1,02 & 9 & 0 \\
\hline Investigaciones Económicas & 0,03 & 3 & 0 & 0,13 & 2 & 0 \\
\hline Información Comercial Española & 0,34 & 6 & 0 & 0,33 & 4 & 0 \\
\hline Revista de Economía Aplicada & 0,005 & 1 & 0 & 0,027 & 1 & 0 \\
\hline Economía Industrial & 0,24 & 8 & 2 & 0,30 & 7 & 0 \\
\hline Revista Europea de Dirección y & 0,07 & 3 & 1 & 0,083 & 1 & 0 \\
Economía de la Empresa & & & & & & 31 \\
\hline
\end{tabular}

Tabla 3: Referencias bibliográficas según idioma y revistas de foros -anglosajón y español-.

\subsection{Investigación sobre DEE en revistas (1990-1997). Análisis para España y Castilla y León}

La revisión de las trece publicaciones consideradas en el periodo 1990-1997 nos ha permitido disponer de una base de datos formada por 245 trabajos, de los que 25 (el 10\% aproximadamente) corresponden a investigadores de CYL. Destaca asimismo el alto número de citas -419 en España y 48 en CYL- para el conjunto de trabajos seleccionados, de manera que podemos decir que a cada trabajo se le asignan, por término medio, 1.7 y 1.92 citas respectivamente. Cabría entonces señalar, como ya lo hacíamos en el apartado anterior, que la investigación en DEE, tanto en el conjunto de España como en nuestra Comunidad Autónoma, no se plasma en trabajos centrados en una única cuestión temática, sino que más bien recurre al análisis del fenómeno de que se trate desde una óptica plural.

Si observamos la distribución temporal de tales trabajos (figura 6), podría señalarse como nota distintiva -ya hablemos del resto de España o de la investigación realizada en CYL- la irregular presencia de los trabajos sobre DEE en las revistas consideradas. Sin embargo, esto no impide reconocer una cierta tendencia de crecimiento, bastante más clara en el resto de España que en CYL.

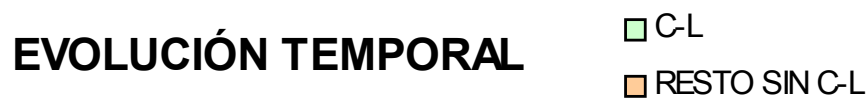

50

40

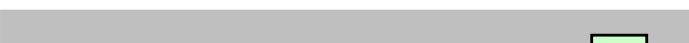

30

$+\square$

20

10

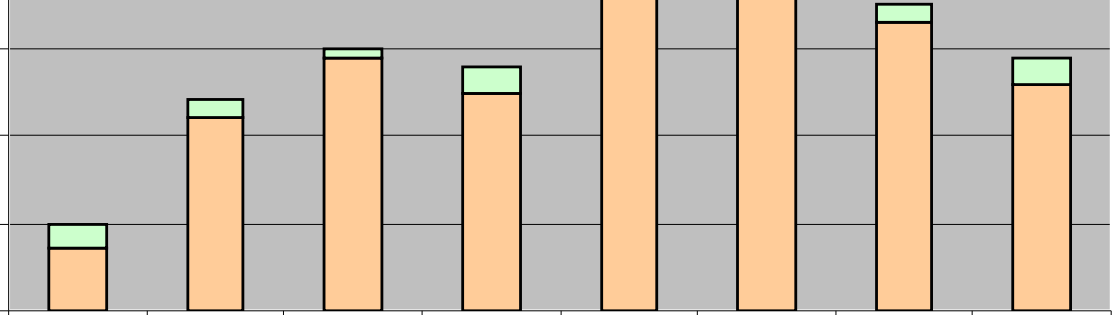


Con una estructura similar a la del apartado anterior, vamos a ver en lo que sigue cómo nos informa la base de datos respecto a revistas analizadas, centros de trabajo y perfil temático. Las referencias bibliográficas no han sido aquí consideradas. También en este caso la óptica de análisis va a ser doble (estática y evolutiva) y la comparación España - Castilla y León permanente.

Revistas analizadas: A la vista de la información disponible en la base de datos, no todas las publicaciones que integran el foro español reconocido en DEE (anexo 1) participan en la misma medida en la difusión de la investigación en dicho campo (figura 7). De entre el conjunto de revistas consideradas, Economía Industrial e Información Comercial Española podrían parecer en principio las más afines a la DEE, en cuanto que son las que durante el periodo considerado publican el mayor porcentaje del total de trabajos sobre estrategia empresarial. Ahora bien, como ya planteábamos en García et al. (1999a), si tenemos en cuenta la periodicidad de cada revista y, en definitiva, el total de trabajos revisados para cada una en el periodo considerado, debemos matizar que, aunque no existe un domicilio fijo para la DEE, las revistas en las que parece contar con mayor peso son: Revista Europea de Dirección y Economía de la Empresa y Dirección y Organización. Éstas no se sitúan en ningún caso entre las más apreciadas. Una participación más ocasional de la DEE parece producirse en Cuadernos Aragoneses de Economía, Revista de Economía Aplicada o Revista Asturiana de Economía. Podemos decir, por tanto, que las revistas españolas con mayor dedicación al campo estratégico son, amén de escasas, medianamente reconocidas por la comunidad científica. Esto hace que los investigadores traten de orientar sus trabajos hacia aquellas publicaciones más reconocidas pero menos centradas en el ámbito estratégico, lo que tiende a mantener la dispersión en la difusión de trabajos de dicho ámbito. Por último, si prestamos atención a las revistas a las que se dirigen los investigadores castellano-leoneses, tenemos que señalar que en Valladolid publican el $45 \%$ de sus trabajos en revistas con elevado reconocimiento o con un mayor peso de la estrategia, en Salamanca lo hacen en un $50 \%$ de los casos y en León en un $83 \%$. Así, ésta última, aún teniendo el menor número de trabajos durante el periodo analizado, ha logrado, al parecer, una adecuada difusión para los mismos.

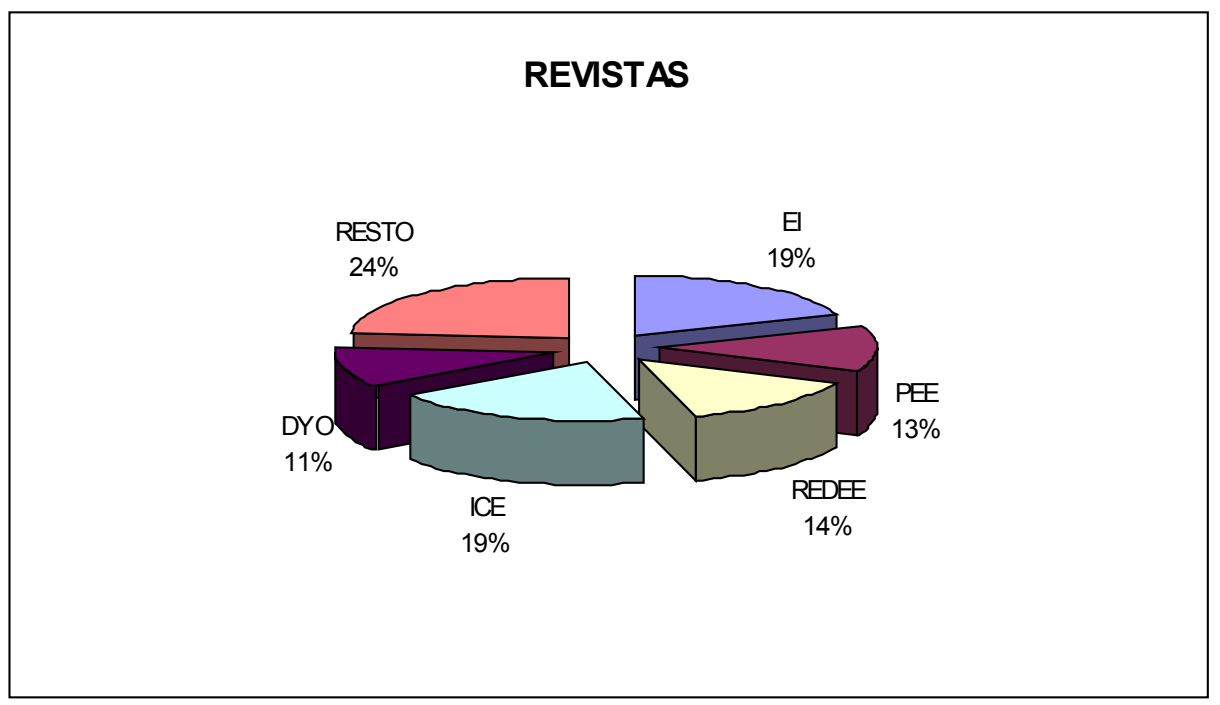

Figura 7: Peso relativo de las revistas analizadas. 
Desde el punto de vista de la trayectoria temporal, podemos decir que 1994, 1995 y 1996 han sido los años más prolíficos a la hora de difundir la investigación sobre estrategia en el foro y también señalar la presencia continua de la estrategia en aquellas revistas que, de acuerdo con la figura 7, cuentan con mayor peso relativo. De este modo, y siempre teniendo en cuenta el año de creación de cada revista, tal continuidad se observa en: Economía Industrial, Información Comercial Española, Papeles de Economía Española, Revista Europea de Dirección y Economía de la Empresa y Dirección y Organización -éstas últimas creadas en 1992-.

Centros de trabajo: Al igual que sucedía en el análisis de la investigación asociada a los congresos, si reparamos en las organizaciones vinculadas a los trabajos revisados, destaca también el elevado porcentaje que los centros universitarios representan sobre el total (figura 8). La distribución por centros de los diferentes trabajos sobre DEE nos permite constatar que la participación de centros no universitarios, con ser minoritaria (en torno a un 17\%), no resulta tan escasa como en el caso de los congresos y que existe un desequilibrio en la participación de las distintas universidades, aunque menos acentuado que para los trabajos en congresos. Así, mientras que a la Universidad de Oviedo le corresponde un $11 \%$ de los trabajos y a la Universidad de Valencia un 8\%, los porcentajes de participación de las universidades castellano-leonesas son los siguientes: un 4\% la Universidad de Valladolid, un 3\% la de Salamanca y un $2 \%$ la de León. La Universidad de Burgos, debido seguramente a su juventud, no tiene participación en la base de datos. Conviene, no obstante, precisar que, por lo que a CYL se refiere, a la Universidad de Valladolid se debe el $44 \%$ de los trabajos incluidos en la base de datos, un $32 \%$ a la Universidad de Salamanca y un $24 \%$ a la de León. Se corrobora así la posición ocupada por la Universidad de Valladolid en lo que a investigación en DEE se refiere. Es necesario insistir, no obstante, en que, para una correcta interpretación de los desequilibrios observados en la participación de las distintas universidades, convendría tener en cuenta la historia más o menos reciente de cada una de ellas o, al menos, de aquellos de sus equipos que se han centrado en DEE. La trayectoria temporal de las tres universidades de CYL con presencia en la base de datos de revistas revela la irregular presencia de tales universidades en las publicaciones del foro español, así como la tardía participación de las universidades de León y Salamanca.

\section{DISTRIBUCIÓN POR CENTROS}

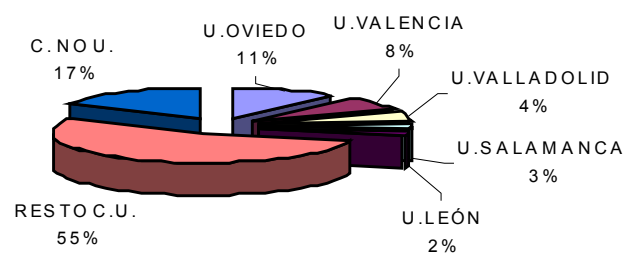

Figura 8: Distribución de los trabajos en revistas por centros. El lugar de CYL.

Perfil temático: Del análisis de la figura 9 se desprenden unos resultados bastante similares a los alcanzados, para el conjunto nacional, en el caso de los congresos. Así, se debe hablar de la existencia de 
un claro desequilibrio en el esfuerzo investigador dedicado a los distintos bloques temáticos que integran la DEE, de manera que mientras el que se centra en la ejecución estratégica sólo representa un $8 \%$, el relativo a objetivos, formulación y elección de la estrategia recoge el $48 \%$ de las citas asociadas. Para los bloques restantes la homogeneidad es aquí bastante evidente, oscilando entre un $14 \%$ y un $15 \%$. Los sujetos que, en mayor grado, centran la atención de los investigadores son los relativos a: cooperación, desarrollo de mercados, objetivos, estrategias genéricas, paradigmas en el desarrollo de la dirección estratégica, caracterización del sector y ventaja competitiva. El perfil temático para CYL presenta ciertas particularidades (figura 10): el bloque relativo a la ejecución estratégica adquiere aquí un peso al que no estamos acostumbrados (13\%); aunque sigue ostentando un claro liderazgo el bloque correspondiente a objetivos, formulación y elección de la estrategia (44\%), la delimitación teórica le sigue los pasos con un $27 \%$ de las citas -mostrando así una clara preocupación del investigador castellano-leonés por el sustrato teórico del campo estratégico-, y, finalmente, el peso del análisis estratégico se ve aquí claramente reducido (un $16 \%$ entre el análisis externo y el interno, contando éste último con sólo un $6 \%$ de las citas). Así pues, mientras que en los trabajos en congresos el investigador castellano-leonés ya ha puesto de manifiesto su cada vez mayor interés por el análisis empresarial interno, en los trabajos difundidos en revistas dicho interés no es aún tan evidente. ¿Será quizá que sí se cumple en el proceso de difusión de la investigación esa secuencia -congresos y después revistas- a la que aludíamos en la introducción de este trabajo? La evidencia es ciertamente débil, por lo que convendría abordarla con más detenimiento en un futuro trabajo. ¿O será simplemente consecuencia del mayor tiempo necesario para la aceptación y publicación de trabajos en las revistas? Nada podemos concluir por tanto a este respecto, al menos por el momento.

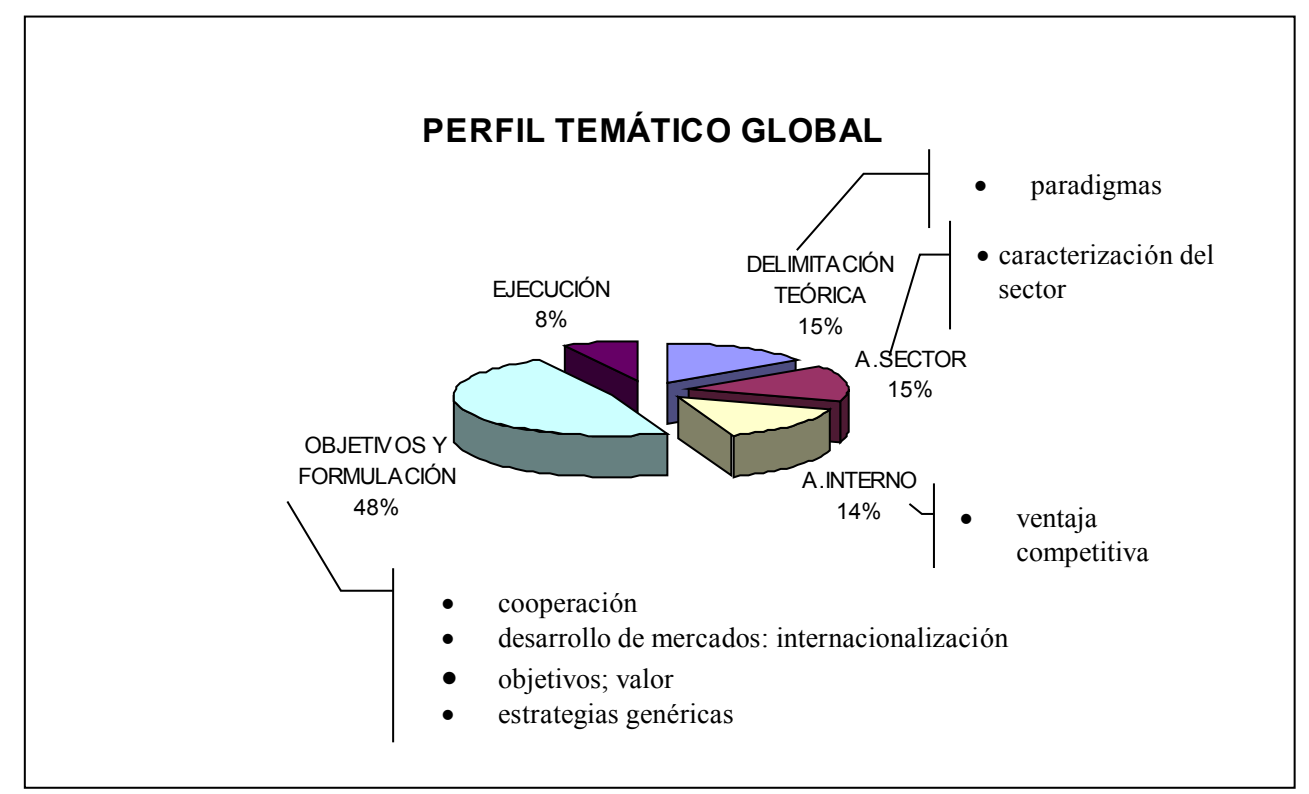

Figura 9: Perfil temático para España en revistas.

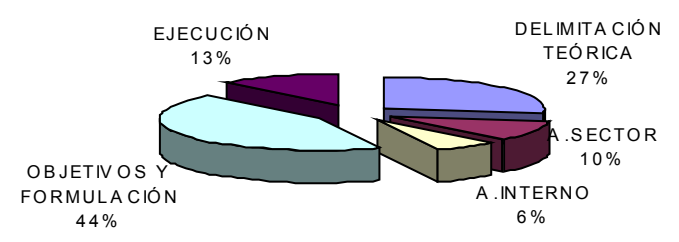


Si ahora consideramos el perfil de cada uno de los centros públicos universitarios de Castilla y León, comprobamos que, al menos en Valladolid y Salamanca, tales particularidades se cumplen. León, sin embargo, cuenta con un perfil propio: delimitación teórica y análisis externo son los bloques que lideran, seguidos de cerca por objetivos y formulación y, después, por el análisis interno.

\section{CONCLUSIONES}

Nos limitaremos aquí a destacar algunas de las conclusiones más importantes que hemos extraído en el desarrollo de esta investigación. Por lo que respecta a los medios de difusión, los investigadores en DEE utilizan preferentemente los foros de debate de dirección y economía de empresa y tienden, en buena medida, a orientar el fruto de sus esfuerzos hacia publicaciones reconocidas -aunque no sean específicas del campo estratégico- antes de acudir a otras más próximas pero con menor reconocimiento, lo que parece que dificultará la consolidación de un domicilio fijo para la estrategia en España. Además, resulta evidente la necesidad de contar con más foros en CYL y el interés de dotar de medios especializados al campo de la estrategia -ya sea en España o en CYL-. No estaría equivocado un respaldo al desarrollo de dichos medios en nuestra Comunidad, a juzgar por el atractivo que parece ejercer el ámbito espacial más próximo -sirva de ejemplo el congreso de economía regional- sobre los investigadores castellano-leoneses. Ahora bien, no se trata tan sólo de contar con medios para difundir la investigación sino que, además, debe lograrse que éstos alcancen unos adecuados niveles de rigor y calidad.

Y, en lo que concierne a la investigación, vamos a señalar una serie de aspectos. Por ejemplo, que, tanto en el conjunto nacional como en CYL, se observa el empleo de una óptica plural en la actividad investigadora en DEE y una mayor preocupación en los últimos tiempos por el análisis estratégico interno ${ }^{17}$ sobre todo en CYL-, lo que se refleja de forma más contundente en la revisión de trabajos difundidos en congresos que en los aparecidos en revistas. Este último aspecto podría deberse al tiempo necesario para publicar en revistas (ciertamente superior a la difusión en congresos) y quizá respaldar la secuencia "congresos-revistas" a la que nos referimos en la introducción del trabajo. No obstante, la evidencia en este sentido es tan débil que convendría insistir, en un trabajo posterior, sobre este sujeto. Además de preocuparse por el análisis estratégico interno, los investigadores castellano-leoneses -especialmente los de Valladolid y Salamanca- han mostrado un interés poco corriente por la ejecución estratégica y la delimitación teórica -que, en el conjunto nacional, parecen haber sido un tanto olvidadas-, lo que podría proporcionarles una posición particular nada desfavorable a la hora de proporcionar conocimiento sobre la DEE a cuantos sujetos lo demanden desde el ámbito social. En cualquier caso, parece claro que, tanto en CYL como en el conjunto nacional, hay todo un conjunto de tópicos que reclaman una mayor atención de los investigadores. Además, el análisis realizado muestra el claro referente de los trabajos anglosajones como soporte teórico de la investigacion española en DEE y, concretamente, el frecuente recurso a la Strategic Management Journal, lo que además parece ser bastante claro en el ámbito de CYL. Por ello no es extraño que la investigación en DEE en nuestro país se aproxime con ciertas garantías a las pautas que marca el mundo anglosajón (García y Santos, 2000b, p.25).

${ }^{17}$ Lo que, como ya hemos indicado, parece lógico por el desarrollo durante los años 90 del enfoque de recursos y capacidades en el marco de la DEE. 
Revista Eletrônica de Ciência Administrativa (RECADM) - ISSN 1677-7387

Faculdade Cenecista de Campo Largo - Coordenação do Curso de Administração v. 2, n. 2, nov./2003 - http://revistas.facecla.com.br/index.php/recadm/

Otros aspectos igualmente detectados serían: la escasa coordinación interuniversitaria en la actividad investigadora $^{18}$-que no es propia únicamente del ámbito castellano-leonés-, pese a que los perfiles temáticos parecen mostrarnos que, al menos desde el punto de vista de los contenidos a abordar, la coordinación sería posible, y, por otro lado, la aún reducida cooperación con investigadores de otros países, lo que es aplicable no sólo a los investigadores de CYL sino a los del conjunto nacional. Algunos rasgos característicos de los investigadores de CYL que convendría valorar y, si es posible, mejorar son: su irregular presencia en los diferentes medios -congresos y revistas- a lo largo del tiempo; una cierta dispersión en la atención prestada a los tópicos de investigación, al menos en el caso de los congresos, o la desigual posición de sus distintas universidades, lo que, sin olvidar su diferente historia, reclama un esfuerzo adicional a algunas de ellas.

Por último, no podemos concluir este trabajo sin reclamar un respaldo aún mayor para los investigadores en general, y los de DEE en particular, ya sea apoyando la coordinación interuniversitaria regional, nacional e internacional-, desarrollando los medios de difusión, o, cómo no, facilitando mayores recursos que posibiliten y mejoren el desarrollo de su tarea. Sin ello, no serán los investigadores los verdaderamente perjudicados sino que lo será la sociedad en su conjunto.

\section{BIBLIOGRAFÍA:}

CASTAGNOS J.C.; BOISSIN, J.P. y GUIEU, G. (1997): "Revues francophones et recherche en stratègie". Économies et Sociétés, Sciences de Gestion, nº 7-8, pp.37-73.

FERNÁNDEZ, Z. (1999): “Presentación”. Investigaciones Europeas de Dirección y Economía de la Empresa, vol. $5, \mathrm{n}^{\circ} 1$, pp.11-20.

FRANKE, R.H., EDLUN, T.W. y OSTER F. (1990): “ The Development of Strategic Management: Journal Quality and Article Impact”. Strategic Management Journal, vol. 11, pp. 243-253.

GARCÍA, Ma T., RODRÍGUEZ, J. y SANTOS, M ${ }^{a}$ V. (1999a): “Una aproximación al estado de la investigación estratégica en España". Ponencia en el Il Workshop Internacional "La organización del futuro en la sociedad de la información: gestión del cambio, recursos humanos y estructura", Universidad de Cádiz, 25-28 de mayo.

GARCÍA, Ma T., RODRÍGUEZ, J. y SANTOS, Ma V. (1999b): "Evaluation of production and diffusion of strategy knowledge. A comparative North American - European balance". $15^{\text {th }}$ EGOS (European Group for Organization Studies) Colloquium, University of Warwick, UK, 4-6 july.

GARCÍA, Ma T., RODRÍGUEZ, J. y SANTOS, Ma V. (2000): "El estado de la Investigación en Dirección Estratégica en España. Una aproximación mediante publicaciones periódicas". Cuadernos de Información Económica, n 156, pp. 80-91.

GARCÍA, $M^{a}$ T. y SANTOS, $M^{a}$ V. (2000a): "La Interdisciplinariedad en la investigación en Dirección Estratégica de la Empresa". Ponencia presentada en el VI Taller de Metodología de Acede, Bayona, Mayo.

GARCÍA, M ${ }^{a}$ T. y SANTOS, M ${ }^{a}$ V. (2000b): "El paralelismo de la investigación estratégica en España con el modelo internacional". Boletín Económico de ICE, n² 2668, pp.15-28.

GUERRAS, L.A.; RUIZ, F.J. y RUIZ, A.V. (1999): "El estado actual de la investigación empírica sobre economía de la empresa: análisis de las publicaciones españolas". Papeles de Economía Española, $\mathrm{n}^{\circ}$ 78-79, pp.302-317.

JOHNSON, G. y SCHOLES, K. (1997): Dirección estratégica. Análisis de la estrategia de las organizaciones. Prentice Hall, Madrid.

MARTINET, A.C. (1993): "Stratégie et pensée complexe". Revue française de gestion, nº 93, mars-avril-mai, pp.64-72.

${ }^{18}$ Aspecto éste que ya se ponía de manifiesto en Guerras et al. (1999, p.309) y en García et al. (2000, pp.87-88). 
MATHÉ, J.C. (1995): "Rétrospectives et Perspectives en Stratégie de l'Entreprise". Économies et Sociétés, Sciences de Gestion, n²1, pp. 31-50.

RUMELT, R.P., SCHENDEL, D.E. y TEECE, D.J. (1994): “Fundamental Issues in Strategy". En Rumelt, R., Schendel, D. y Teece, D. (edtrs.): Fundamental Issues in Strategy. A Research Agenda. Harvard Business School Press, Boston, Mass., pp. 9-47.

SALAS, V. (1995): "La economía de la empresa en la universidad pública española". Revista Española de Financiación y Contabilidad, vol. XXIV, nº 84, pp.815-839.

SANTOS, Ma V., GARCÍA, Ma T. y RODRÍGUEZ, J. (1999a): "Comparative international analysis of strategic management journals: The character of Spanish journals". En Kantarelis, D. (ed.): Business \& Economics for the $21^{\text {st }}$ Century. Volume III, pp.197-211.

SANTOS, Ma V., GARCÍA, Ma T. y RODRÍGUEZ, J. (1999b): "Evolución de la investigación española sobre estrategia en el foro de publicaciones periódicas”. IX Congreso de ACEDE, Burgos, 12-14 Septiembre.

SNOW, C. y THOMAS, J.(1994): "Field research methods in strategic management: contributions to theory building and testing". Journal of Management Studies, 31, 4, pp. 457-480.

TEECE, D., PISANO G, y SHUEN, A. (1997): “Dynamic Capabilities and Strategic Management”. Strategic Management Journal, vol. 18, 7, pp. 509-533.

VENTURA, J. (1996): Análisis dinámico de la estrategia empresarial: un ensayo interdisciplinar. Servicio de Publicaciones, Universidad de Oviedo. 
Revista Eletrônica de Ciência Administrativa (RECADM) - ISSN 1677-7387 Faculdade Cenecista de Campo Largo - Coordenação do Curso de Administração v. 2, n. 2, nov./2003 - http://revistas.facecla.com.br/index.php/recadm/

ANEXO 1

\begin{tabular}{|l|}
\hline \multicolumn{1}{|c|}{ FORO ESPAÑOL DE PUBLICACIONES APRECIADAS EN DEE } \\
\hline BOLETIN DE ESTUDIOS ECONÓMICOS (BEE) \\
INFORMACIÓN COMERCIAL ESPAÑOLA (ICE) \\
ECONOMÍA INDUSTRIAL (EI) \\
\hline ESIC-MARKET \\
INVESTIGACIONES ECONÓMICAS (IE) \\
\hline CUADERNOS ARAGONESES DE ECONOMÍA (CAE) \\
PAPELES DE ECONOMÍA ESPANOLA (PEE) \\
HARVARD DEUSTO BUSINESS REVIEW (HDBR) \\
REVISTA EUROPEA DE DIRECCIÓN Y ECONOMÍA DE LA EMPRESA (REDEE) \\
\hline DIRECCIÓN Y ORGANIZACIÓN (DYO) \\
REVISTA DE ECONOMÍA APLICADA (REA) \\
REVISTA ASTURIANA DE ECONOMÍA (RAE) \\
INVESTIGACIONES EUROPEAS DE DIRECCIÓN Y ECONOMÍA DE LA EMPRESA \\
(IEDYEE) \\
CUADERNOS DE ECONOMÍA Y DIRECCIÓN DE EMPRESA (CEDE) \\
\hline
\end{tabular}


ANEXO 2

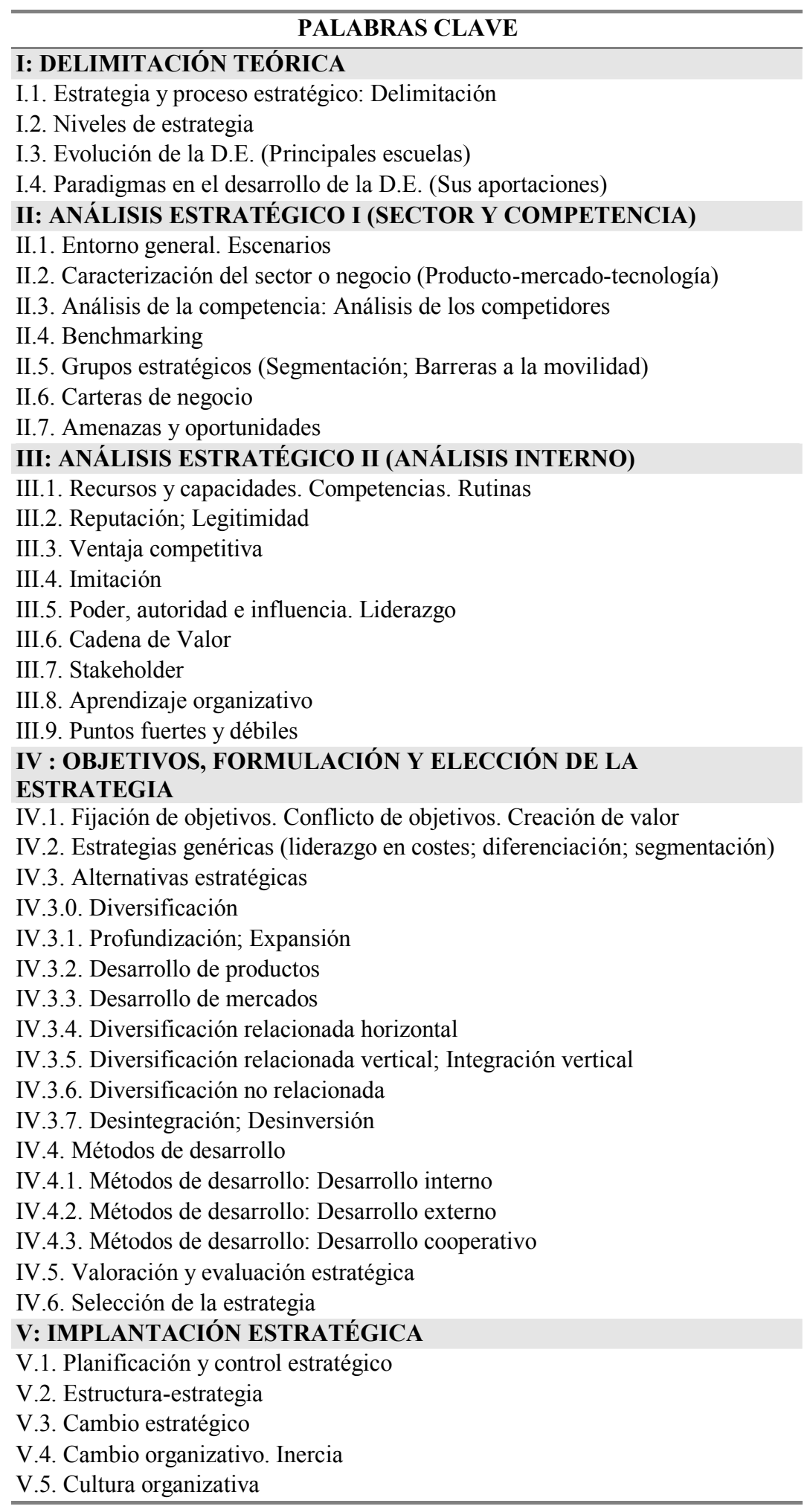

